\title{
Solid and aqueous magnetoliposomes as nanocarriers for a new potential drug active against breast cancer
}

\author{
Ana Rita O. Rodrigues, ${ }^{1}$ Pedro M. F. Mendes, ${ }^{1}$ Pedro M. L. Silva, ${ }^{1}$ V. A. Machado, ${ }^{2}$ \\ Bernardo G. Almeida, ${ }^{1,3}$ J. P. Araújo, ${ }^{4}$ Maria-João R. P. Queiroz, ${ }^{2}$ Elisabete M. S. \\ Castanheira, ${ }^{1}$ Paulo J. G. Coutinho ${ }^{1,3^{*}}$ \\ ${ }^{1}$ Centre of Physics (CFUM), University of Minho, Campus de Gualtar, 4710-057 Braga, Portugal \\ ${ }^{2}$ Centre of Chemistry (CQ-UM), University of Minho, Campus de Gualtar, 4710-057 Braga, Portugal \\ ${ }^{3}$ QuantaLab, University of Minho, Campus de Gualtar, 4710-057 Braga, Portugal \\ ${ }^{4}$ IFIMUP/IN - Instituto de Nanociência e Nanotecnologia, R. Campo Alegre, 4169-007 Porto, Portugal
}

\section{Statistical summary:}

Total number of words (with references): 6834

Total number of words (without references): 4796

Figures: 7

Tables: 1

Supplementary material available

\section{Keywords:}

iron oxide nanoparticles; aqueous magnetoliposomes; solid magnetoliposomes; antitumor drug; dual cancer therapy

\section{*Corresponding Author:}

Paulo J. G. Coutinho

Centre of Physics and QuantaLab, University of Minho,

Campus de Gualtar,

4710-057 Braga, Portugal

Phone: +351 253 604321;

Fax: +351253604061

pcoutinho@fisica.uminho.pt 


\begin{abstract}
Iron oxide nanoparticles, with diameters around $12 \mathrm{~nm}$, were synthesized by coprecipitation method. The magnetic properties indicate a superparamagnetic behavior with a coercive field of 9.7 Oe and a blocking temperature of $118 \mathrm{~K}$. Both aqueous and solid magnetoliposomes containing magnetite nanoparticles have sizes below $150 \mathrm{~nm}$, suitable for biomedical applications. Interaction between both types of magnetoliposomes and models of biological membranes was proven. A new antitumor compound, a diarylurea derivative of thienopyridine, active against breast cancer, was incorporated in both aqueous and solid magnetoliposomes, being mainly located in the lipid membrane. A promising application of these magnetoliposomes in oncology is anticipated, allowing a combined therapeutic approach, using both chemotherapy and magnetic hyperthermia.
\end{abstract}

\title{
1. Introduction
}

The potential of magnetic nanoparticles for biomedical applications has been recognized, as they offer major advantages due to their unique size and physicochemical properties [1]. Nanoparticles with superparamagnetic behavior are preferred for these purposes, as they exhibit a strong magnetization only when an external magnetic field is applied [2-4]. Liposomes entrapping magnetic nanoparticles (magnetoliposomes) can be guided and localized in the therapeutic site by external magnetic field gradients and used in cancer therapy by hyperthermia [5-8]. Moreover, liposomes revealed advantageous in overcoming issues of solubility, pharmacokinetics, toxicity and in vivo stability and toxicity of other delivery systems [9-11]. Magnetoliposomes have been used as a chemotherapy alternative through magnetically-controlled drug delivery and thermotherapy [12-15]. Iron oxide nanoparticles $\left(\mathrm{Fe}_{3} \mathrm{O}_{4}, \gamma-\mathrm{Fe}_{2} \mathrm{O}_{3}\right)$ have been widely used due to their proven biocompatibility and low toxicity [16-20]. Magnetoliposomes containing magnetite nanoparticles have been used in MRI [21], environmental applications [22], thermo/chemotherapy [23] and treatment of other diseases [24]. These nanosystems take advantage of the very large saturation magnetization reported for magnetite nanoparticles [16,17,22], higher than the one observed for nickel ferrite [25] and manganese ferrite nanoparticles [26]. 
Thienopyridines including their diarylurea derivatives have shown different biological activities, namely as antitumoral agents [27] and receptor tyrosine kinase inhibitors [28]. Recently, a strong VEGFR-2 inhibition activity, with very low $\mathrm{IC}_{50}$ values (on the order of $10 \mathrm{nM}$ ) was reported for a series of new arylurea derivatives of thienopyridines [29]. Also, a potent antiproliferative activity on two human breast cancer cell lines of distinct types, a hormone-dependent MCF-7 and hormone independent MDA-MB-231 cell lines, was described for this type of compounds [30].

In this work, both aqueous and dry magnetoliposomes based on magnetite nanoparticles were prepared by soft templating methods and characterized. The interaction between the prepared magnetoliposomes and models of cell membranes (giant unilamellar vesicles, GUVs) was evaluated using FRET (Förster Resonance Energy Transfer).

A new diarylurea derivative of thienopyridine 1 (Figure 1), with potent activity against breast cancer cell lines [30], was incorporated in both types of magnetoliposomes. Compound 1 exhibits a strong antitumor activity in MCF-7 and MDA-MB-231 breast tumor cell lines ( $\mathrm{IC}_{50}$ of $1.2 \mu \mathrm{M}$ and $5.0 \mu \mathrm{M}$, respectively [30]), the latter being the most aggressive and difficult to treat. Specifically, the therapy against the highly metastatic MDA-MB-231 cancer cells could strongly benefit from the synergistic effect of chemotherapy and magnetic hyperthermia. Therefore, these studies are of main importance for the development of a combined thermo/chemotherapy for breast cancer.

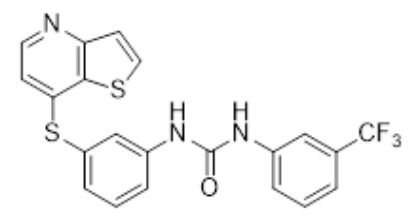

Figure 1. Structure of the arylurea derivative of thienopyridine.

\section{Experimental}

All the solutions were prepared using spectroscopic grade solvents and ultrapure water (Milli-Q grade).

\subsection{Magnetite nanoparticles preparation}

\subsubsection{Coprecipitation method}

Magnetite nanoparticles (NPs) were prepared by a coprecipitation method in aqueous solution, using fresh $\mathrm{Fe}^{2+}$ and $\mathrm{Fe}^{3+}$ solutions in a 1:2 stoichiometry [31]. A 0.15 M iron 
(II) sulfate solution $\left(\mathrm{FeSO}_{4} \cdot 7 \mathrm{H}_{2} \mathrm{O}\right)$ and a $0.3 \mathrm{M}$ iron (III) sulfate solution $\left(\mathrm{Fe}_{2}\left(\mathrm{SO}_{4}\right)_{3} \cdot 9 \mathrm{H}_{2} \mathrm{O}\right)$ were used. $27 \mu \mathrm{L}$ of each solution were added to $2 \mathrm{~mL}$ of water and $200 \mu \mathrm{L}$ of sodium hydroxide $(2.3 \mathrm{M})$ and the mixture was kept at $80^{\circ} \mathrm{C}$ for 2 hours, in absence of oxygen. The magnetite nanoparticles formed through the reaction:

$$
\mathrm{Fe}^{2+}+\mathrm{Fe}^{3+}+8 \mathrm{OH}^{-} \rightarrow \mathrm{Fe}_{3} \mathrm{O}_{4}+4 \mathrm{H}_{2} \mathrm{O}
$$

After the synthesis, the precipitated nanoparticles were washed several times with ultrapure water, recovered by magnetic decantation and redispersed in the initial volume of water.

\subsubsection{Reverse coprecipitation method}

In the reverse coprecipitation method, only one iron solution is used [32,33]. $121 \mu \mathrm{L}$ of $\mathrm{NaOH}$ solution $(50 \%, 18.9 \mathrm{M})$ were added to $10 \mathrm{~mL}$ of ultrapure water to adjust $\mathrm{pH}$ to 13. A fresh $0.2 \mathrm{M}$ solution of iron (II) sulfate was prepared. $5 \mathrm{~mL}$ of the latter solution were added, drop by drop, to the $\mathrm{NaOH}$ solution, under magnetic stirring. On an alternative procedure, the $5 \mathrm{~mL}$ of $\mathrm{Fe}^{2+}$ solution were added, also drop by drop, in five portions of $1 \mathrm{~mL}$ each, each addition separated by 10 minutes. In both cases, the total concentration of iron was $66 \mathrm{mM}$. The reaction was kept for one hour at room temperature.

The precipitated nanoparticles were then washed several times with ultrapure water and recovered by magnetic decantation.

\subsection{Preparation of magnetoliposomes}

\subsubsection{Aqueous magnetoliposomes}

Aqueous magnetoliposomes (AMLs) are formed when the magnetic nanoparticles are encapsulated in liposomes. Egg yolk phosphatidylcholine (Egg-PC), from Sigma-Aldrich, was used for lipid vesicle formation. A $10 \mathrm{mM} \mathrm{Egg-PC}$ solution in ethanol was injected, under vigorous vortexing, to an aqueous solution of nanoparticles (ethanolic injection method [34,35]). After encapsulation, the ferrofluid was washed with water and purified by magnetic decantation and centrifugation to remove all the non-encapsulated NPs.

\subsubsection{Solid magnetoliposomes}


DPPC (1,2-Dipalmitoyl-sn-glycero-3-phosphocholine) and DOPG (1,2-Dioleoyl-snglycero-3-phospho-rac-(1-glycerol) sodium salt) from Avanti Polar Lipids, was used to produce a double lipid layer around the magnetite nanoparticles, following the procedure previously developed by us for nickel ferrite nanoparticles [25]. First, $760 \mu \mathrm{L}$ of the synthesized magnetite NPs (in 2.1.1) were centrifuged. Then, the deposited particles were redispersed in $10 \mu \mathrm{L}$ water in an ultrasonicator for one minute at $189 \mathrm{~W}$, and $3 \mathrm{~mL}$ of chloroform were added to the aqueous dispersion of NPs. After vigorous agitation, $165 \mu \mathrm{L}$ of a $20 \mathrm{mM}$ solution of DPPC (or DOPG) were added under vortexing, to form the first lipid layer of solid magnetoliposomes (SMLs). The particles sedimented using a small magnet and the supernatant was discarded. The particles were then washed twice by magnetic decantation with pure water, in order to remove the lipid that was not attached to the NPs. The second lipid layer was then formed by the injection of $165 \mu \mathrm{L}$ of lipid ( $20 \mathrm{mM})$, under vortexing, in a $3 \mathrm{~mL}$ aqueous dispersion of the particles with the first lipid layer. The resulting SMLs were then washed twice, purified with pure water by centrifugation and redispersed in $3 \mathrm{~mL}$.

Compound 1 was incorporated into aqueous magnetoliposomes by the co-injection method, as already described [26]. In solid magnetoliposomes, compound 1 was incorporated by injection of an ethanolic solution together with the formation of the second lipid layer [26].

\subsection{Preparation of Giant Unilamellar Vesicles (GUVs)}

Soybean lecithin ( $L$ - $\alpha$-Phosphatidylcholine), from Sigma-Aldrich, was used for GUVs preparation, using a procedure previously described [36,37]. A film of soybean lecithin was obtained by evaporation under an ultrapure nitrogen stream of a $1 \mathrm{mM}$ lipid solution. This film was incubated with $20 \mu \mathrm{L}$ of water at $45^{\circ} \mathrm{C}$ for 45 minutes. Then, 3 $\mathrm{mL}$ of $0.1 \mathrm{M}$ glucose solution was added, and the resulting mixture was again incubated at $37^{\circ} \mathrm{C}$.

\subsection{Spectroscopic measurements}

\subsubsection{General methods}

Absorption spectra were recorded in a Shimadzu UV-3101PC UV-vis-NIR spectrophotometer. Fluorescence measurements were performed using a Fluorolog 3 spectrofluorimeter, equipped with double monochromators in both excitation and 
emission and a temperature controlled cuvette holder. Fluorescence spectra were corrected for the instrumental response of the system.

\subsubsection{FRET measurements}

The interaction of magnetoliposomes with models of biological membranes (GUVs) was evaluated by Förster Resonance Energy Transfer (FRET). FRET efficiency, $\Phi_{\text {RET }}$, defined as the proportion of donor molecules that have transferred their excess energy to acceptor molecules, was calculated through donor emission quenching, by taking the ratio of the donor integrated fluorescence intensities in the presence of acceptor and in the absence of acceptor [38]. The distance between donor and acceptor molecules was determined through the FRET efficiency (equation 1),

$$
r=R_{0} \cdot\left[\frac{1-\Phi_{\mathrm{RET}}}{\Phi_{\mathrm{RET}}}\right]^{1 / 6}
$$

where $R_{0}$ is the Förster radius (critical distance), that can be obtained by the spectral overlap, $\mathrm{J}(\lambda)$, between the donor emission and the acceptor absorption, according to equations (2) and (3) (with $R_{0}$ in $\AA, \lambda$ in $\mathrm{nm}, \varepsilon_{\mathrm{A}}(\lambda)$ in $\mathrm{M}^{-1} \mathrm{~cm}^{-1}$ ), [38]

$$
\begin{gathered}
R_{0}=0.2108\left[k^{2} \Phi_{\mathrm{D}}^{0} n^{-4} J(\lambda)\right]^{1 / 6} \\
J(\lambda)=\int_{0}^{\infty} I_{D}(\lambda) \varepsilon_{A}(\lambda) \lambda^{4} d \lambda
\end{gathered}
$$

where $k^{2}=2 / 3$ is the orientational factor assuming random orientation of the dyes, $\Phi_{D}^{0}$ is the fluorescence quantum yield of the donor in the absence of energy transfer, $n$ is the refraction index of the medium, $\mathrm{I}_{\mathrm{D}}(\lambda)$ is the fluorescence spectrum of the donor normalized so that $\int_{0}^{\infty} I_{D}(\lambda) d \lambda=1$, and $\varepsilon_{\mathrm{A}}(\lambda)$ is the molar absorption coefficient of the acceptor.

FRET assays were employed to confirm the formation of the second lipid bilayer in the solid magnetoliposomes (SMLs). For that purpose, the rhodamine B labeled lipid Rhodamine-DHPE (1,2-dipalmitoyl-sn-glycero-3-phosphoethanolamine- $N$-(lissamine rhodamine B sulfonyl) (ammonium salt) (from Avanti Polar Lipids, structure in Figure S1 of the Supplementary Material) was included the first lipid layer, while the nitrobenzoxazole labeled lipid NBD-C6-HPC (1-palmitoyl-2-\{6-[(7-nitro-2-1,3benzoxadiazol-4-yl)amino]hexanoyl $\}$-sn-glycero-3-phosphocholine) (from Avanti Polar Lipids, structure also in Figure S1) was included in the second lipid layer. 
For the study of the interaction of magnetoliposomes with GUVs, the former were labeled with both NBD-C 6 -HPC and the hydrophobic probe Nile Red (from Fluka, structure in Figure S1).

The fluorescence quantum yield, $\Phi_{s}$, of the energy donor (in both cases, the dye NBD) in magnetoliposomes was determined by the standard method (equation 4) $[39,40]$,

$$
\Phi_{s}=\frac{A_{r} F_{s} n_{s}^{2}}{A_{s} F_{r} n_{r}^{2}} \Phi_{r}
$$

where $A$ is the absorbance at the excitation wavelength, $F$ the integrated emission area and $n$ the refraction index of the solvents used. Subscripts refer to the reference (r) or sample (s). The absorbance at the excitation wavelength was always lower than 0.1 to avoid the inner filter effects. The NBD-C 6 -HPC molecule intercalated in lipid membranes was used as reference, $\Phi_{\mathrm{r}}=0.32$ at $25^{\circ} \mathrm{C}$, as reported by Invitrogen [41].

\subsubsection{Fluorescence anisotropy measurements}

The steady-state fluorescence anisotropy, $r$, is calculated by

$$
r=\frac{I_{\mathrm{VV}}-G I_{\mathrm{VH}}}{I_{\mathrm{VV}}+2 G I_{\mathrm{VH}}}
$$

where $I_{\mathrm{VV}}$ and $I_{\mathrm{VH}}$ are the intensities of the emission spectra obtained with vertical and horizontal polarization, respectively (for vertically polarized excitation light), and $G=I_{\mathrm{HV}} / I_{\mathrm{HH}}$ is the instrument correction factor, where $I_{\mathrm{HV}}$ and $I_{\mathrm{HH}}$ are the emission intensities obtained with vertical and horizontal polarization (for horizontally polarized excitation light).

\subsection{Structural characterization}

Scanning electron microscopy (SEM) images of magnetite nanoparticles and solid magnetoliposomes were recorded using a Scanning Electron Microscope FEI - Nova 200 NanoSEM. In the case of SMLs, a negative staining was employed. For that, a $2 \%$ aqueous solution of ammonium molybdate tetrahydrate was prepared. Then, $20 \mu \mathrm{L}$ of sample and $20 \mu \mathrm{L}$ of staining solution were mixed and a drop of this mixture was placed onto a Formvar grid, held by tweezers. After 20 seconds, almost all the solution was removed with filter paper and left dry. 
The processing of SEM images was performed using ImageJ software. It consisted in enhancing local contrast followed by automatic local thresholding and particle analysis. The area of each particle allowed an estimation of its size. The resulting histograms were fitted to Gaussian distributions.

Liposomes mean diameter and size distribution (polydispersity index) were measured using a Dynamic Light Scattering (DLS) equipment (NANO ZS Malvern Zetasizer) at $25^{\circ} \mathrm{C}$, using a He-Ne laser of $\lambda=632.8 \mathrm{~nm}$ and a detector angle of $173^{\circ}$. Five independent measurements were performed for each sample.

X-Ray Diffraction (XRD) analyses were performed using a conventional Philips PW 1710 diffractometer, operating with $\mathrm{Cu} \mathrm{K}_{\alpha}$ radiation, in a Bragg-Brentano configuration.

\subsection{Magnetic measurements}

Magnetic measurements were performed at room temperature in a Superconducting Quantum Interference Device (SQUID) magnetometer (Quantum Design MPMS5XL), using applied magnetic fields up to $5.5 \mathrm{~T}$.

The temperature dependence of the magnetization was measured in the temperature range from $5 \mathrm{~K}$ to $380 \mathrm{~K}$. The curves were obtained by initially cooling the sample under an applied magnetic field of $\mathrm{H}=100 \mathrm{Oe}$ (field cooled, FC) and then measuring its magnetization with increasing temperature (applied field of $\mathrm{H}=50 \mathrm{Oe}$ ). Subsequently, after reaching $380 \mathrm{~K}$, the sample was re-cooled, this time with no applied magnetic field (zero-field-cooled, ZFC) and the magnetization measurements were again performed with increasing temperature, under the same magnetic field of $\mathrm{H}=50$ Oe. From the behavior of the FC and ZFC curves, the blocking temperature $\left(T_{B}\right)$ of the superparamagnetic nanoparticles can be obtained [42]. The magnetization hysteresis loop measurements were made by fixing the temperature and measuring the magnetization at a series of different applied magnetic fields. This type of study gives information about the maximum magnetization and the degree at which the sample remains magnetized when the applied field is removed, and how easily the sample magnetization can be reversed, the so-called coercive field.

\subsection{Nanoparticles encapsulation efficiency in AMLs}

The nanoparticles encapsulation efficiency in aqueous magnetoliposomes (AMLs) was estimated from the spectrophotometric determination of iron (III) content, through the 
formation of a phenylfluorone complex sensitized with Triton X-100 [43]. To obtain iron (III) from the magnetoliposomes, the latter were digested by heating at $500^{\circ} \mathrm{C}$ overnight, to remove all the biological components. Then, $1 \mathrm{~mL}$ of concentrated nitric acid (from Sigma-Aldrich) was added and the sample was heated to $80^{\circ} \mathrm{C}$ for 2 hours. The temperature was then raised to $150^{\circ} \mathrm{C}$ for $48 \mathrm{~h}$, and the $\mathrm{pH}$ was increased until 5.5 by successive cycles of addition and evaporation of ultrapure water (Milli-Q grade). Finally, $1 \mathrm{~mL}$ of water was added to the digested sample and, after ultrasonication for one hour, the iron (III) content of magnetoliposomes was released.

For the spectrophotometric measurements, the standard addition method was employed.

$100 \mu \mathrm{L}$ of the digested sample and $2 \times 10^{-5} \mathrm{M}, 3 \times 10^{-5} \mathrm{M}, 4 \times 10^{-5} \mathrm{M}, 5 \times 10^{-5} \mathrm{M}$ or $6 \times 10^{-5} \mathrm{M}$ of iron (III) stock solution were added to $1.6 \times 10^{-4} \mathrm{M}$ phenylfluorone and $4 \times 10^{-3} \mathrm{M}$ Triton X-100 solutions. The $\mathrm{pH}$ was then adjusted to 9 using $0.05 \mathrm{M}$ borax buffer [43]. A calibration curve for the determination of iron (III) concentration was previously obtained. Three independent measurements were performed and standard deviations (SD) were calculated. The encapsulation efficiency, $E E(\%)$, of iron oxide nanoparticles (NPs) in AMLs was determined by equation:

$E E(\%)=\frac{(\text { total amount of NPs }- \text { amount of non-encapsulated NPs })}{\text { total amount of NPS }} \times 100$

\section{Results and discussion}

\subsection{Characterization of magnetite nanoparticles}

\subsubsection{Scanning electron microscopy (SEM)}

SEM images of the nanoparticles prepared by coprecipitation in aqueous solution (preparation method described in 2.1.1) revealed generally spherical nanoparticles uniform in size, with a size distribution of $11.6 \pm 1.6 \mathrm{~nm}$ (Figure 2). 


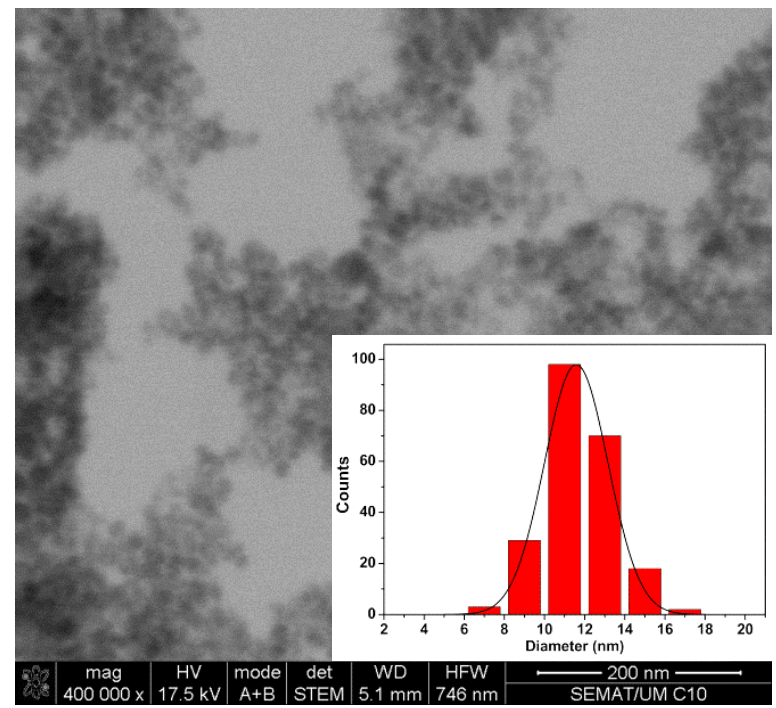

Figure 2. SEM image of magnetite nanoparticles prepared by coprecipitation method in aqueous solution. Inset: Particles size histogram and fitting to a Gaussian distribution.

Magnetite nanoparticles obtained by reverse coprecipitation method in aqueous solution (protocol 2.1.2.) exhibit larger sizes and a higher polydispersity (Figure 3), with some "fibrous" structures in the case of adding iron (II) in five times, with 10 minutes intervals (Figure 3B). For nanoparticles prepared adding all $\mathrm{Fe}^{2+}$ at once, a size distribution of $21.7 \pm 3.8 \mathrm{~nm}$ was obtained, while a diameter of $20.9 \pm 2.7 \mathrm{~nm}$ was determined for the nanoparticles obtained with addition of $\mathrm{Fe}^{2+}$ in five portions.

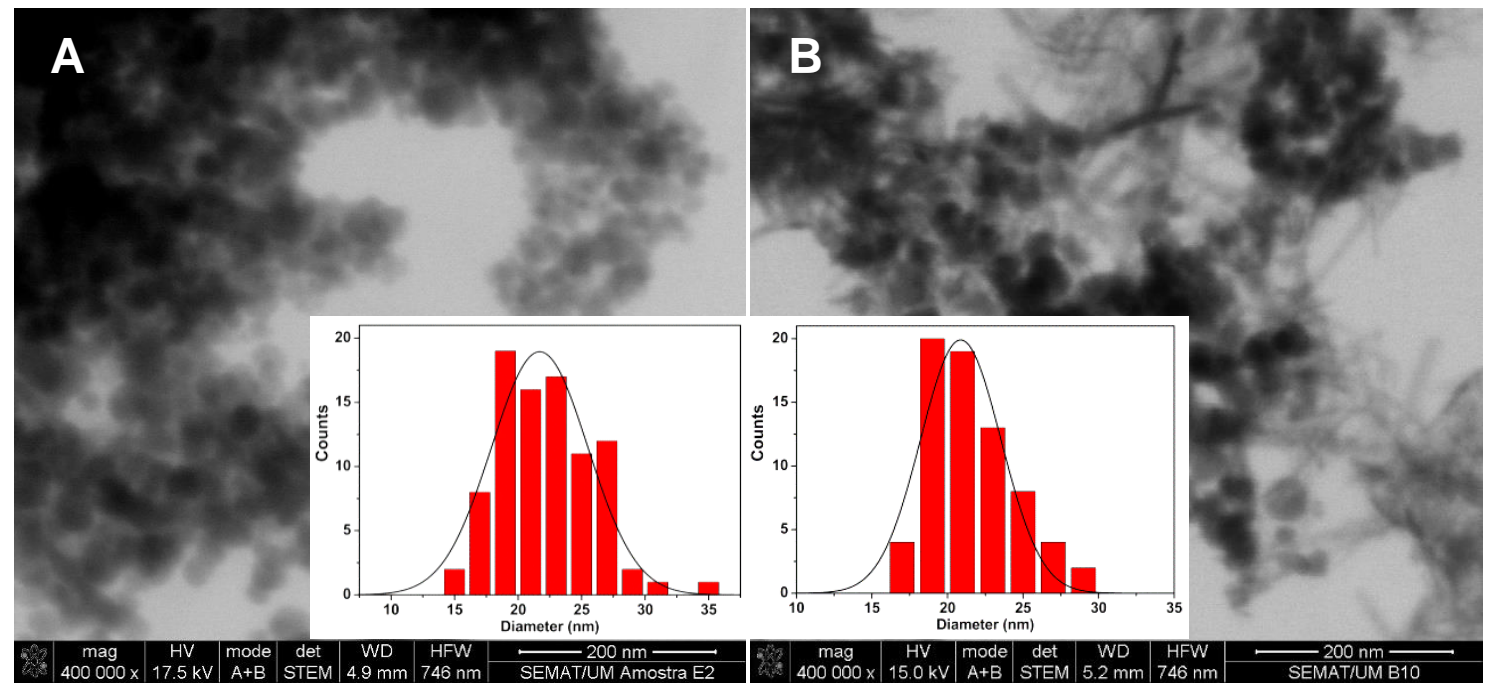

Figure 3. SEM images of magnetite nanoparticles prepared by reverse coprecipitation method in aqueous solution. A: Addition of all $\mathrm{Fe}^{2+}$ at once; $\mathbf{B}$ : Addition of $\mathrm{Fe}^{2+}$ in five portions, with 10 minutes intervals. Insets: Particles size histograms and fitting to Gaussian distributions.

According to these results, magnetite nanoparticles prepared by coprecipitation method in aqueous solution (Figure 2, corresponding to protocol 2.1.1) were chosen for further 
characterization and for the preparation of aqueous and solid magnetoliposomes, considering their small size, low polydispersity and low degree of aggregation.

\subsubsection{XRD measurements}

The synthesis of magnetite NPs consisting of a pure crystalline phase was confirmed by XRD analysis (Figure 4), since all their characteristic peaks (CIF 9000926), marked by their indices, are observed. The absence of high temperature thermal treatment results in a significant amorphous background in the XRD pattern. Rietveld analysis using FullProf software [44] was performed by modeling the background as a linear interpolation between a set of points at constant scattering angles, but with fitted intensities. A good fit was possible by using preferred orientation correction at $\left(\begin{array}{lll}1 & 1 & 0\end{array}\right)$ plane, as it can be observed in the residuals (Figure 4B). This correction is based on the March function [45], equation 7,

$$
\left(r^{2} \cos ^{2} \alpha+\frac{\sin ^{2} \alpha}{r}\right)^{-3 / 2}
$$

where, for a platy habit, $\alpha$ is the acute angle between the scattering vector and the normal to the crystallites. The obtained fitted value indicates a degree of preferred orientation of $(1-r)=0.41$. Preferred orientation of crystallites within a powder sample was also reported for the case of $\mathrm{Cd}$ doped $\mathrm{ZnO}$ [46] and $\mathrm{Mn}$ doped $\mathrm{ZnO}$ [47]. The obtained reduced $\chi^{2}$ was 1.20 , with an $\mathrm{R}_{\mathrm{F}}$ value of $7.49 \%$, and the estimated size of the $\mathrm{Fe}_{3} \mathrm{O}_{4}$ crystallites based on the Scherrer formula was $5.6 \mathrm{~nm}$. The value of $\mathrm{R}_{\mathrm{F}}$ improves to $5.67 \%$ if the overall isothermal factor, $\mathrm{B}_{\text {over, }}$, is optimized, but an unreasonable value of -2.95 is obtained. A summary of the results of Rietveld analysis is shown in Table S1 of the Supplementary Material. 


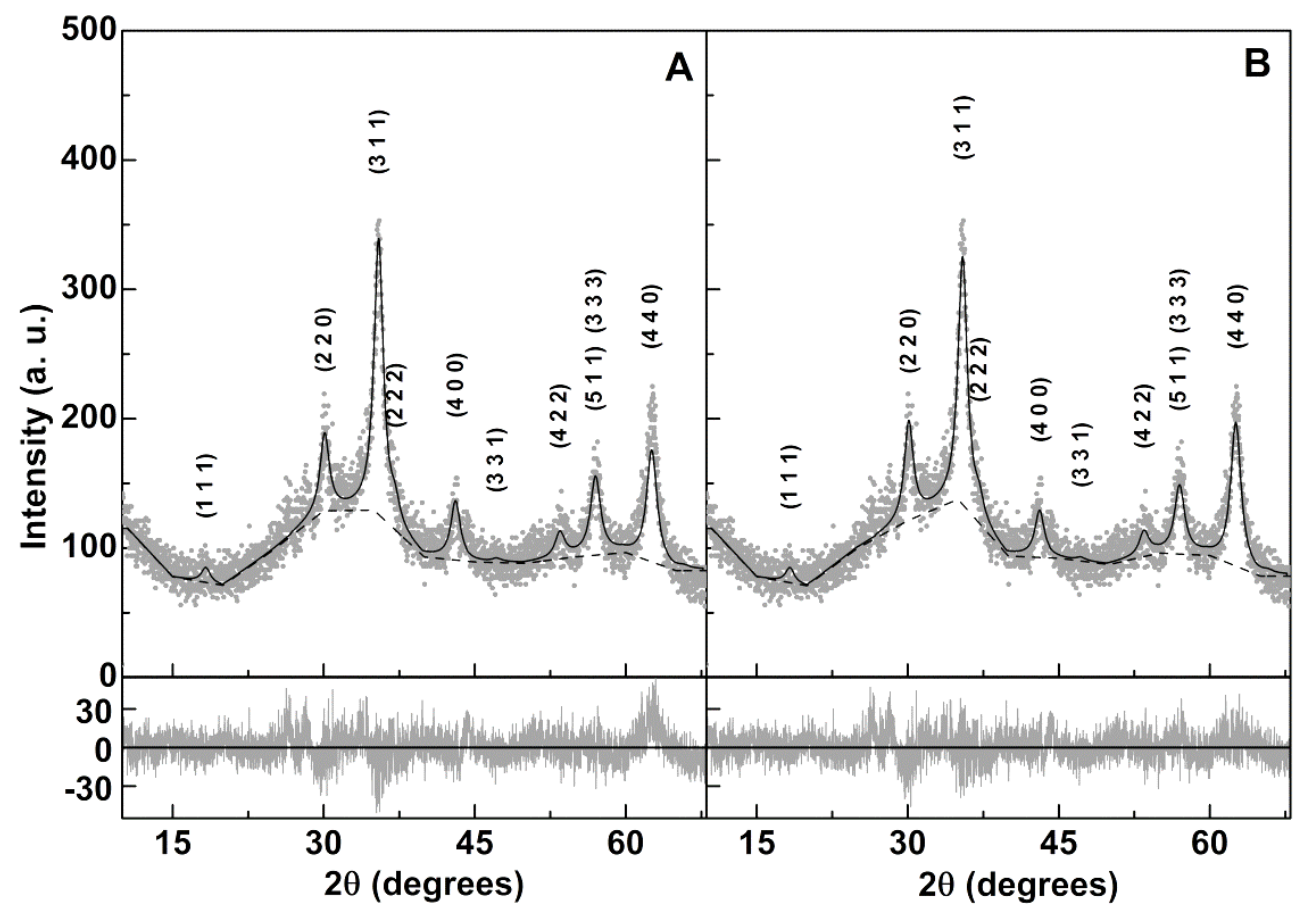

Figure 4. Rietveld analysis of XRD patterns of the sample, with (A) and without $(\mathbf{B})$ preferred orientation correction at (1 110$)$ plane.

\subsubsection{Magnetic Properties}

The magnetic properties of the nanoparticles synthesized by coprecipitation method (Figure 2) were characterized by measuring their corresponding magnetic hysteresis loop, which shows the relationship between the induced magnetic moment and the applied magnetic field $(H)$. Figure 5 shows the hysteresis cycle measured on the prepared magnetite nanoparticles. A magnetic squareness value (ratio between the residual magnetization, $M_{\mathrm{r}}$, and the saturation magnetization, $M_{\mathrm{s}}$ ) of 0.023 was obtained, indicating that the synthesized nanoparticles are superparamagnetic at room temperature $[42,48]$. Typical magnetic properties were observed, with a very slight hysteresis, a saturation magnetization of $62 \mathrm{emu} / \mathrm{g}$ (Figure 5A) and a coercive field of 9.7 Oe (Figure $5 \mathrm{~B})$. Reported values for saturation magnetization of $\mathrm{Fe}_{3} \mathrm{O}_{4}$ nanoparticles typically vary from 50 to $80 \mathrm{emu} / \mathrm{g}$, depending on their size [49-56]. 

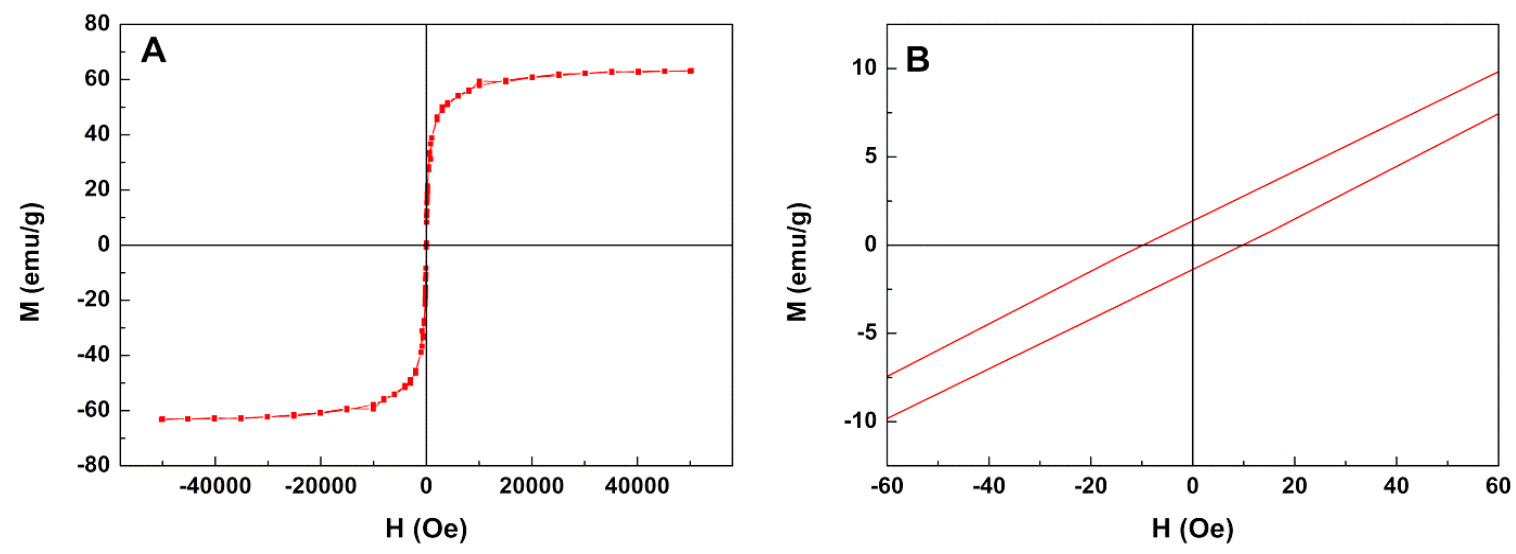

Figure 5. A: Magnetization hysteresis cycle of magnetite nanoparticles at $\mathrm{T}=300 \mathrm{~K}$. B: Enlargement of the hysteresis loop of Figure 5A, in the low field region.

Previous reports found negligible coercivity values for magnetite NPs of $8 \mathrm{~nm}$ size, while for nanoparticles of $12 \mathrm{~nm}$ a coercivity of 18 Oe was observed [49,50]. It was also shown that $\mathrm{Fe}_{3} \mathrm{O}_{4}$ nanoparticles with $16 \mathrm{~nm}$ and a coercive field of 27 Oe were able to produce heat with high efficiency in hyperthermia applications [49]. The low coercive field and the very reasonable saturation magnetization of the synthesized $\mathrm{Fe}_{3} \mathrm{O}_{4}$ nanoparticles point to a promising utility for hyperthermia applications. In fact, the inductive heating capability of magnetic nanoparticles, under AC magnetic field, is directly proportional to the area of the hysteresis cycle [55] and thus decreases as the coercive field is reduced, but increases as the saturation magnetization gets higher. A low coercive field in superparamagnetic particles is important to avoid extensive permanent agglomeration. Its negative effect on inductive heating capability can however be compensated by the occurrence of small clusters of magnetic nanoparticles, that originate an additional and significant heating capacity, enough to reach therapeutic temperatures [57].

The superparamagnetic particles are characterized by distinct behaviors of the temperature dependence of zero-field-cooling and field-cooling magnetization curves. Starting from the low temperatures on the ZFC curve, as the temperature increases the blocked magnetic moments align with the applied measuring magnetic field, leading to an initial increase of the sample magnetization. However, as soon as thermal fluctuations are able to allow the moments to overcome the magnetic anisotropy energy barrier, the thermal randomization of the intraparticles magnetic moments produces a subsequent decrease of the magnetization curve, with increasing temperature. The zero-field-cooling curve peak corresponds to the blocking temperature, $\mathrm{T}_{\mathrm{B}}$ [42], and here a value of $\mathrm{T}_{\mathrm{B}}=118 \mathrm{~K}$ was obtained for $\mathrm{Fe}_{3} \mathrm{O}_{4}$ NPs (Figure S2 in Supplementary 
Material). On the FC curve, the magnetic moments were initially forced to be aligned with the magnetic field imposed during cooling. This then gives a significant overall magnetization at low temperatures, as seen on the FC curve (Figure S2), which

monotonically decreases with increasing temperature. Above the blocking temperature, NPs present superparamagnetic behavior and the ZFC and FC curves show the same temperature dependence of the magnetization, which can be approached by Curie law. At lower temperature, the ZFC and FC curves separate each other indicating that some nanoparticles are thermally blocked and are not able to completely align with the applied field. Here, the nanoparticles show ferromagnetic behavior, as thermal fluctuations are not enough to randomize the intraparticles magnetic moments.

For an ensemble of superparamagnetic particles above the blocking temperature, the magnetization can be described by the Langevin function [42]

$\mathrm{M}(\mathrm{H}, \mathrm{T})=N \mu L\left(\frac{\mu H}{k_{B} T}\right)=N \mu\left(\operatorname{coth}\left(\frac{\mu H}{k_{B} T}\right)-\frac{k_{B} T}{\mu H}\right)$

where $\mu$ is the particle magnetic moment, $k_{\mathrm{B}}$ is the Boltzmann constant, $T$ is the absolute temperature, $N$ is the number of particles per volume and $H$ is the applied magnetic field.

The magnetization hysteresis cycle was fitted to equation (8), assuming a spherical shape and using the density of $\mathrm{Fe}_{3} \mathrm{O}_{4} \mathrm{NPs}, 5.18 \mathrm{~g} / \mathrm{cm}^{3}$ [56]. Diameters around $5.4 \mathrm{~nm}$ were estimated, the high $R^{2}$ value $\left(R^{2}=0.9934\right)$ indicating a good fit to the experimental data. This particle diameter is in excellent agreement with the one obtained from XRD analysis (section 3.1.2., Table S1).

\subsection{Characterization of magnetoliposomes and interaction with model membranes}

Two types of magnetoliposomes were prepared, aqueous magnetoliposomes (AMLs) and solid magnetoliposomes (SMLs) and the interaction with GUVs (giant unilamellar vesicles), used as models of cell membranes, was investigated.

\subsubsection{Aqueous magnetoliposomes}

Aqueous magnetoliposomes were obtained by ethanolic injection of the lipids in the aqueous ferrofluid. This method is very advantageous for hydrophobic drugs (with very limited solubility in water, as is the case of compound 1), which can be loaded in 
(magneto)liposomes by coinjection. The NPs encapsulation efficiency in these liposomes was calculated from the spectrophotometric determination of iron (III), following the procedure described in [43]. The encapsulation efficiency, obtained from three independent assays, was $E E(\%) \pm S D(\%)=46 .(9) \pm 15$. This result compares well with the ones reported for the extrusion method by Sabate et al. ( $E E=19 \%$ in the worst case) [58] and Ferreira et al. (EE=66\% in their best formulation) [59] and proves the suitability of the ethanolic injection method when hydrophobic drugs are considered to be loaded in AMLs. It is also probable that the encapsulation efficiency is dependent on the initial amount of ferrofluid used, as reported $[58,59]$.

The size distribution of aqueous magnetoliposomes (AMLs) of egg phosphatidylcholine containing magnetite nanoparticles was measured by DLS. A hydrodynamic diameter of $112 \pm 19 \mathrm{~nm}$ was determined (Figure S3 in Supplementary Material). This size is slightly larger than those obtained for AMLs of the same lipid containing nickel ferrite [25] or manganese ferrite [26] nanoparticles.

The possibility of membrane fusion between aqueous magnetoliposomes (AMLs) and giant unilamellar vesicles (GUVs), used as models of cell membranes, was evaluated by FRET. The labeled lipid NBD-C ${ }_{6}$-HPC and the lipid probe Nile Red were both included in AMLs, NBD acting as the energy donor and Nile Red as the energy acceptor [60]. The solvatochromic and hydrophobic dye Nile Red [61-65] exhibits a red shift in emission maximum in polar solvents. Moreover, owing to its capability to establish H-bonds with protic solvents, Nile Red fluorescence in water is very weak, exhibiting a maximum wavelength around $660 \mathrm{~nm}$ [65].

When both fluorophores are incorporated in magnetoliposomes, an efficient energy transfer process is observed, exciting only the donor NBD (Figure S4 in Supplementary Material). Two fluorescence bands are observed, the first $\left(\lambda_{\max }=535 \mathrm{~nm}\right)$ corresponding to NBD emission and the second to Nile Red fluorescence $\left(\lambda_{\max }=630 \mathrm{~nm}\right)$, arising from the energy transfer of excited NBD molecules to Nile Red. If the magnetoliposomes interact with GUVs, a larger membrane will be formed by fusion. Consequently, an increase in the donor-acceptor distance will occur with a corresponding decrease in the energy transfer efficiency from NBD to Nile Red. This is observed in Figure S4, through the increase in donor emission and the decrease of acceptor fluorescence, proving membrane fusion between both systems. The assay was performed for AMLs of Egg-PC (Figure S4-A), which is in the liquid-crystalline phase at room temperature and AMLs of DPPC (Figure S4-B), which is in the gel phase at $25{ }^{\circ} \mathrm{C}$. Aqueous 
magnetoliposomes of DPPC/cholesterol were also recently obtained by Ferreira et al. [59]. Our results in Figure S4 confirm that fusion with GUVs occurs for both AMLs of Egg-PC and DPPC. This result is similar to those previously observed with aqueous magnetoliposomes of Egg-PC containing different types of magnetic nanoparticles $[25,26,60]$.

\subsubsection{Solid magnetoliposomes}

The SMLs were synthesized by the procedure previously developed by us using nickel ferrite and manganese ferrite nanoparticles [25,26]. SMLs containing iron oxide nanoparticles and with lipid bilayers of unsaturated lipids were previously obtained by Meledandri et al. [21], using the lipids DOPG and DOPC (18:1 PC), and by Zhang et al. [22], using soybean phosphatidylcholine. In this work, clusters of magnetic nanoparticles were covered with the phospholipid dipalmitoyl phosphatidylcholine (DPPC or 16:0 PC), which possesses two saturated hydrocarbon chains and a melting transition temperature of $41{ }^{\circ} \mathrm{C}$ [66]. For comparison, SMLs containing a lipid bilayer of the unsaturated lipid dioleoyl phosphatidylglycerol (DOPG or 18:1 PG) were also prepared.

The formation of a DPPC bilayer around the nanoparticles was confirmed by FRET between NBD (donor) and Rhodamine (acceptor), using a methodology reported in previous works $[25,26]$. Briefly, the labeled lipid Rhodamine-DHPE was included in the first (inner) lipid layer and the labeled lipid NBD- $\mathrm{C}_{6}-\mathrm{HPC}$ was included in the second (outer) lipid layer. A $\Phi_{\mathrm{D}}^{0}$ value of 0.11 for $\mathrm{NBD}-\mathrm{C}_{6}-\mathrm{HPC}$ in magnetite SMLs was determined. An energy transfer efficiency of $75 \%$ was obtained, corresponding to a donor-acceptor distance of $3.43 \mathrm{~nm}$ (equations 1-3). This result evidences the formation of the double lipid layer in DPPC SMLs, considering the usual thickness of a biological membrane (7 to $9 \mathrm{~nm}[67]$ ).

Dynamic Light Scattering measurements revealed that the DOPG and DPPC SMLs are approximately monodisperse (Figures S5 and S6 in Supplementary Material). Hydrodynamic diameters of $136 \pm 32 \mathrm{~nm}$ and of $127 \pm 28 \mathrm{~nm}$ were obtained, respectively, for DOPG and DPPC SMLs, revealing that these nanosystems are suitable for drug delivery applications, due to their size below $150 \mathrm{~nm}$ and low polydispersity.

The structure of solid magnetoliposomes does not present an inner water pool and, therefore, solid magnetoliposomes can be observed by SEM microscopy. SEM image 
(Figure 6) of DPPC SMLs containing $\mathrm{Fe}_{3} \mathrm{O}_{4}$ nanoparticles shows spherical structures with $c a .123 \mathrm{~nm}$ diameter, in accordance with DLS results. These SMLs are structurally similar to the ones first reported by De Cuyper and Joniau [68], but the preparation method is much simpler and expedite, as there is neither the need for an initial step of coating of the magnetic nanoparticles with lauric acid, nor the dialysis procedure, that can take up to two days.

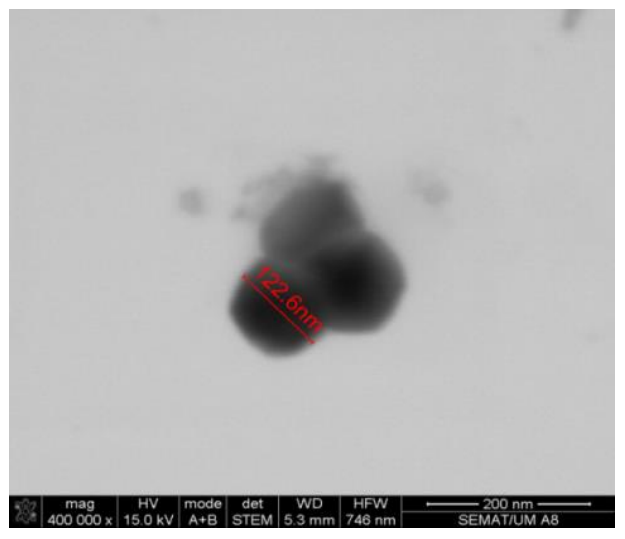

Figure 6. SEM image of solid magnetoliposomes of the lipid DPPC containing magnetite nanoparticles.

\subsection{Incorporation of the antitumor compound 1 in magnetoliposomes}

The thienopyridine derivative 1 (Figure 1) presents very low growth inhibitory concentration values in MCF-7 breast adenocarcinoma cell line and in the aggressive MDA-MB-231 breast tumor cell line [30]. For this reason, compound $\mathbf{1}$ was incorporated in both AMLs and SMLs containing magnetite nanoparticles and the incorporation was monitored by fluorescence emission, taking advantage of the intrinsic fluorescence of this potential drug. Figure 7 shows the emission spectra of 1 in AMLs, SMLs and liposomes (without magnetic nanoparticles and with the same concentration of compound). It is possible to observe a quenching effect of the fluorescence emission by the magnetic nanoparticles, much more pronounced in SMLs, indicating the incorporation of the thienopyridine derivative in both AMLs and SMLs, as previously observed for another thienopyridine derivative in magnetoliposomes containing manganese ferrite NPs [26].

Fluorescence anisotropy (steady-state) values for compound $\mathbf{1}$ are presented on Table 1. For comparison, the anisotropy of the compound was measured in the highly viscous solvent glycerol ( $\eta=0.9934$ Pa.s at room temperature [69]), with much larger viscosity than the one usually reported for lipid membranes (between 0.1 and 0.2 Pa.s [70,71]). 
The measured steady-state anisotropies clearly indicate that this antitumor compound is mainly located in the lipid membrane of liposomes. These values are significantly lower than the one measured in glycerol, mainly due to the distinct viscosities of both types of media (despite possible differences in excited-state lifetimes).

Table 1. Steady-state fluorescence anisotropy $(r)$ values for antitumor compound $\mathbf{1}$ in liposomes, aqueous magnetoliposomes (AMLs) and solid magnetoliposomes (SMLs).

\begin{tabular}{cccc}
\hline & Lipid & Temperature & $\boldsymbol{r}$ \\
\hline \multirow{4}{*}{ Liposomes } & Egg-PC & $25^{\circ} \mathrm{C}$ & 0.182 \\
\cline { 2 - 4 } & \multirow{2}{*}{ DPPC } & $25^{\circ} \mathrm{C}$ & 0.251 \\
& & $55^{\circ} \mathrm{C}$ & 0.157 \\
\cline { 2 - 4 } & DOPG & $25^{\circ} \mathrm{C}$ & 0.061 \\
\hline \multirow{3}{*}{ AMLs } & Egg-PC & $25^{\circ} \mathrm{C}$ & 0.185 \\
\cline { 2 - 4 } & \multirow{2}{*}{ DPPC } & $25^{\circ} \mathrm{C}$ & 0.258 \\
& & $55^{\circ} \mathrm{C}$ & 0.147 \\
\cline { 2 - 4 } SMLs & DOPG & $25^{\circ} \mathrm{C}$ & 0.056 \\
& \multirow{2}{*}{ DPPC } & $25^{\circ} \mathrm{C}$ & 0.169 \\
\cline { 2 - 4 } Glycerol & & $55^{\circ} \mathrm{C}$ & 0.112 \\
\hline
\end{tabular}

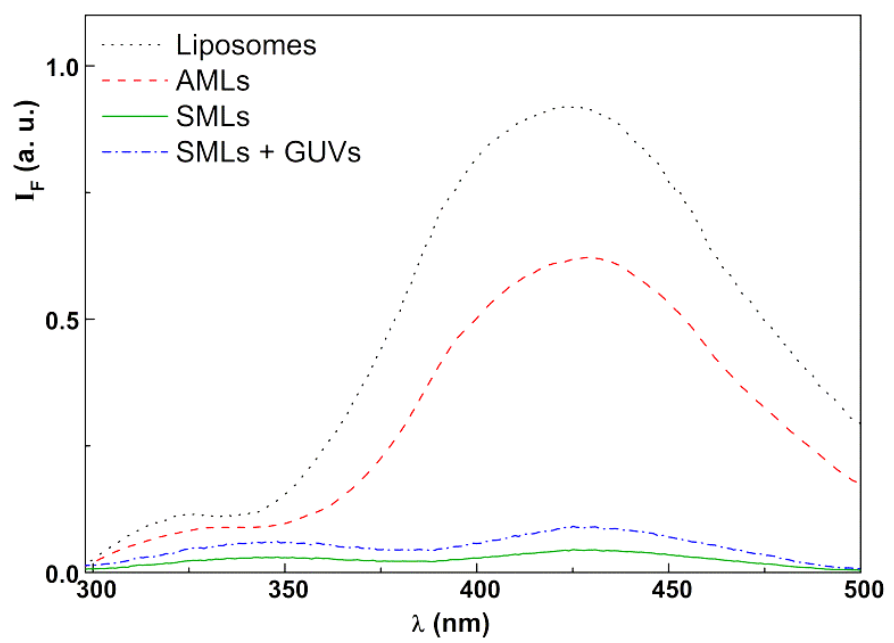

Figure 7. Fluorescence spectra $\left(\lambda_{\text {exc }}=290 \mathrm{~nm}\right)$ of compound $1\left(3 \times 10^{-6} \mathrm{M}\right)$ in liposomes and magnetoliposomes (AMLs and SMLs) of DPPC containing magnetite nanoparticles, and in SMLs after interaction with GUVs.

Comparing the anisotropy values at $25^{\circ} \mathrm{C}$ and $55^{\circ} \mathrm{C}$ in DPPC-based systems, it must be noted that an increase of the steady-state anisotropy is predicted from a diminution of the excited-state lifetime, according to Perrin equation [38]. Upon a rise of temperature, 
the excited-state lifetime is expected to decrease, due to the increment of the nonradiative deactivation pathways, mainly the rate constant for internal conversion $S_{1} \rightarrow S_{0}$. As the anisotropy values decrease strongly at higher temperature (Table 1), this behavior can be attributed to a decrease of the rotational correlation time of the fluorescent compound. This is a result of the diminution of membrane microviscosity upon changing from the gel to the liquid-crystalline phase of the lipid DPPC $\left(\mathrm{T}_{\mathrm{m}}=\right.$ $\left.41{ }^{\circ} \mathrm{C}[66]\right)$. The lipid DOPG is highly fluid at room temperature [72], justifying very low anisotropy values of the compound in liposomes and magnetoliposomes of this lipid. Egg lecithin is a natural mixture of phosphatidylcholines (mainly 16:0 PC, 18:0 PC and 18:1 PC [73]), this composition justifying lower anisotropy values than those of DPPC at room temperature, due to the presence of the unsaturated lipid 18:1 PC.

Comparing liposomes and magnetoliposomes, it can be observed that anisotropy values for this antitumor compound in AMLs and SMLs are, in general, similar to those determined in liposomes of the same lipids. In DPPC SMLs, the anisotropy values are lower than in DPPC liposomes or AMLs. This result points to a deeper location of the compound in SMLs membrane, as it was reported that microviscosity decreases from the interface to the interior of the membrane $[74,75]$. This justifies the strong quenching of compound fluorescence in SMLs. Together, these results confirm that this potential antitumor drug is fully incorporated in both types of magnetoliposomes, located mainly in the lipid bilayer and experiencing changes in fluidity between the rigid gel phase $\left(25^{\circ} \mathrm{C}\right)$ and the liquid-crystalline phase $\left(55^{\circ} \mathrm{C}\right)$ in the case of DPPC.

Interaction of SMLs entrapping compound $\mathbf{1}$ with model membranes was also investigated. Comparing compound emission before and after interaction with GUVs (Figure 7), an unquenching effect is detected upon interaction, confirming membrane fusion of SMLs with GUVs. Therefore, the magnetoliposomes here prepared are promising as carriers for this antitumor compound active against breast cancer. Based on the experimental results, we anticipate that both aqueous and solid drug-loaded magnetoliposomes have potential as therapeutic agents in future applications of chemo/thermotherapy of cancer.

\section{Conclusions}

In this work, magnetite nanoparticles with size around $12 \mathrm{~nm}$ were synthesized by coprecipitation method. $\mathrm{Fe}_{3} \mathrm{O}_{4}$ NPs exhibit superparamagnetic properties, with 
maximum magnetization of $62 \mathrm{emu} / \mathrm{g}$ at $5 \mathrm{~T}$ applied field, a coercivity of $9.7 \mathrm{Oe}$ and a blocking temperature of $118 \mathrm{~K}$. Both aqueous and solid magnetoliposomes containing magnetite nanoparticles, with sizes below $150 \mathrm{~nm}$ and low polydispersity, were prepared and their interaction with model membranes by fusion was demonstrated.

A new antitumor compound active against breast cancer was successfully incorporated in both AMLs and SMLs, which is a promising result for the application of these nanocarriers in dual oncological therapy for breast cancer, using both hyperthermia and chemotherapy.

\section{Acknowledgements}

This work was supported by the Portuguese Foundation for Science and Technology (FCT) in the framework of the Strategic Funding of CF-UM-UP (UID/FIS/04650/2013) and of CQUM (UID/QUI/00686/2013). FCT, POPH-QREN and FSE are acknowledged for the PhD grants of A.R.O. Rodrigues (SFRH/BD/90949/2012) and V.A. Machado (SFRH/BD/77373/2011) and for financial support to MAP-Fis Joint Doctoral Programme.

\section{References}

[1] S. Mornet, S. Vasseur, F. Grasset, E. Duguet, Magnetic nanoparticle design for medical diagnosis and therapy, J. Mater. Chem. 14 (2004) 2161-2175.

[2] A. S. Lubbe, C. Bergemann, J. Brock, D. G. McClure, Physiological aspects in magnetic drug-targeting, J. Magn. Magn. Mater. 194 (1999) 149-155.

[3] D. Frascione, C. Diwoky, G. Almer, P. Opriessnig, C. Vonach, K. Gradauer, G. Leitinger, H. Mangge, R. Stollberger, R. Prassl, Ultrasmall superparamagnetic iron oxide (USPIO)based liposomes as magnetic resonance imaging probes, Int. J. Nanomedicine 7 (2012) 2349-2359.

[4] S. Dandamudi, R. B. Campbell, The drug loading, cytotoxicity and tumor vascular targeting characteristics of magnetite in magnetic drug targeting, Biomaterials 28 (2007) 4673-4683.

[5] S. Dandamudi, R. B. Campbell, Development and characterization of magnetic cationic liposomes for targeting tumor microvasculature, Biochim. Biophys. Acta 1768 (2007) 427438 .

[6] S. Takemori, K. Tazawa, H. Nagae, I. Yamashita, H. Kato, T. Kasagi, M. Maeda, T. Honda, M. Fujimaki, A study of DDS in hyperthermia: inductive heating with use of dextran magnetite (DM), Drug Deliv. Syst. 6 (1991) 465-470. 
[7] A. Jordan, P. Wust, H. Fähling, W. John, A. Hinz, R. Felix, Inductive heating of ferrimagnetic particles and magnetic fluids: physical evaluation of their potential for hyperthermia, Int. J. Hyperthermia 9 (1993) 51-68.

[8] A. Hervault, N. T. K. Thanh, Magnetic nanoparticle-based therapeutic agents for thermochemotherapy treatment of cancer, Nanoscale 6 (2014) 11553-11573.

[9] G. Poste, C. Cucana, A. Raz, P. Bugelski, R. Kirsh, I. J. Fidler, Analysis of the fate of systemically administered liposomes and implications for their use in drug delivery, Cancer Res. 24 (1982) 1412-1422.

[10] G. Gregoriadis, Engineering liposomes for drug delivery: progress and problems, Trends Biotechnol. 13 (1995) 527-537.

[11] T. L. Andresen, S. S. Jensen, K. Jørgensen, Advanced strategies in liposomal cancer therapy: problems and prospects of active and tumor specific drug release, Prog. Lipid Res. 44 (2005) 68-97.

[12] H.-J. Weinmann, W. Ebert, B. Misselwitz, H. Schmitt-Willich, Tissue-specific MR contrast agents, Eur. J. Radiol. 46 (2003) 33-44.

[13] N. Nuytten, M. Hakimhashemi, T. Ysenbaert, L. Defour, J. Trekker, S. J. Soenen, P. Van der Meeren, M. Cuyper, PEGylated lipids impede the lateral diffusion of adsorbed proteins at the surface of (magneto)liposomes, Colloids Surf. B: Biointerfaces 80 (2010) 227-231.

[14] U. I. Tromsdorf, N. C. Bigall, M. G. Kaul, O. T. Bruns, M. S. Nikolic, B. Mollwitz, R. A. Sperling, R. Reimer, H. Hohenberg, W. J. Parak, S. Forster, U. Beisiegel, G. Adam, H. Weller, Size and surface effects on the MRI relaxivity of manganese ferrite nanoparticle contrast agents, Nano Lett. 7 (2007) 2422-2427.

[15] E. Amstad, J. Kohlbrecher, E. Müller, T. Schweizer, M. Textor, E. Reimhult, Triggered release from liposomes through magnetic actuation of iron oxide nanoparticle containing membranes, Nano Lett. 11 (2011) 1664-1670.

[16] R. Hiergeist, W. Andrä, N. Buske, R. Hergt, I. Hilger, U. Richter, W. Kaiser, Application of magnetite ferrofluids for hyperthermia, J. Magn. Magn. Mater. 201 (1999) 420-422.

[17] P. Tartaj, M. P. Morales, S. Veintemillas-Verdaguer, T. González-Carreño, J. C. Serna, The preparation of magnetic nanoparticles for applications in biomedicine, J. Phys. D Appl. Phys. 36 (2003) R182-R197.

[18] J. Ramos, J. Forcada, Surfactant-free miniemulsion polymerization as a simple synthetic route to a successful encapsulation of magnetite nanoparticles, Langmuir 27 (2011) 72227230 .

[19] C. Gonçalves, Y. Lalatonne, L. Melro, G. Badino, M. F. M. Ferreira, L. David, C.F.G.C. Geraldes, L. Motte, J. A. Martins, F. M. Gama, New dextrin nanomagnetogels as contrast agents for magnetic resonance imaging, J. Mater. Chem. B 1 (2013) 5853-5864.

[20] US National Institutes of Health, University of Edinburgh, "Cell tracking using superparamagnetic particles of iron oxide (SPIO) and magnetic resonance imaging (MRI) a pilot study", Clinical trial, University of Edinburgh, UK, 2009.

[21] C. J. Meledandri, T. Ninjbadgar, D. F. Brougham, Size-controlled magnetoliposomes with tunable magnetic resonance relaxation enhancements, J. Mater. Chem. 21 (2011) 214-222.

[22] S. Zhang, H. Niu, Y. Zhang, J. Liu, Y. Shia, X. Zhang, Y. Cai, Biocompatible phosphatidylcholine bilayer coated on magnetic nanoparticles and their application in the 
extraction of several polycyclic aromatic hydrocarbons from environmental water and milk samples, J. Chromatogr. A 1238 (2012) 38-45.

[23] A. Hardiansyah, L.-Y. Huang, M.-C. Yang, T.-Y. Liu, S.-C. Tsai, C.-Y. Yang, C.-Y. Kuo, T.-Y. Chan, H.-M. Zou, W.-N. Lian, C.-H. Lin, Magnetic liposomes for colorectal cancer cells therapy by high-frequency magnetic field treatment, Nanoscale Res. Lett. 9 (2014) article 497.

[24] S. García-Jimeno, E. Escribano, J. Queralt, J. Estelrich, Magnetoliposomes prepared by reverse-phase followed by sequential extrusion: characterization and possibilities in the treatment of inflammation, Int. J. Pharmaceut. 405 (2011) 181-187.

[25] A. R. O. Rodrigues, I. T. Gomes, B. G. Almeida, J. P. Araújo, Elisabete M. S. Castanheira, Paulo J. G. Coutinho, Magnetic liposomes based on nickel ferrite nanoparticles for biomedical applications, Phys. Chem. Chem. Phys. 17 (2015) 18011-18021.

[26] A. R. O. Rodrigues, J. M. F. Ramos, I. T. Gomes, B. G. Almeida, J. P. Araújo, M.-J. R. P. Queiroz, Paulo J. G. Coutinho, Elisabete M. S. Castanheira, Magnetoliposomes based on manganese ferrite nanoparticles as nanocarriers for antitumor drugs, RSC Advances 6 (2016) 17302-17313.

[27] I. Hayakama, R. Shioya, T. Agatsuma, H. Furukawa, Y. Sugano, Thienopyridine and Benzofuran derivatives as potent anti-tumor agents possessing different structureactivity relationship, Bioorg. Med. Chem. 14 (2004) 3411-3414.

[28] H. R. Heyman, R. R. Frey, P. F. Bousquet, G. A. Cunha, M. D. Moskey, A. A. Ahmed, N. B. Soni, P. A. Marcotte, L. J. Pease, K. B. Glaser, M. Yates, J. J. Bouska, D. H. Albert, C. L. Black Schaefer, P. J. Dandliker, K. D. Stewart, P. Rafferty, S. K. Davidsen, M. R. Michaelides, M. L. Curtin, Thienopyridine urea inhibitors of KDR kinase, Bioorg. Med. Chem. Lett. 17 (2007) 1246-1249.

[29] V. A. Machado, D. Peixoto, R. Costa, H. J. C. Froufe, R. C. Calhelha, R. M. V. Abreu, I. C. F. R. Ferreira, R. Soares, M.-J. R. P. Queiroz, Synthesis, antiangiogenesis evaluation and molecular docking studies of 1-aryl-3-[(thieno[3,2-b]pyridin-7-ylthio)phenyl]ureas: Discovery of a new substitution pattern for type II VEGFR-2 Tyr kinase inhibitors, Bioorg. Med. Chem. 23 (2015) 6497-6509.

[30] V. A. Machado, D. Peixoto, M.-J. R. P. Queiroz, R. Soares, Antiangiogenic 1-aryl-3-[3(thieno[3,2-b]pyridin-7-ylthio)phenyl]ureas inhibit MCF-7 and MDA-MB-231 human breast cancer cell lines through PI3K/Akt and MAPK/Erk pathways, J. Cell. Biochem. 117 (2016) 2791-2799.

[31] I. Martínez-Mera, M. E. Espinosa-Pesqueira, R. Pérez-Hernández, J. Arenas-Alatorre, Synthesis of magnetite $\left(\mathrm{Fe}_{3} \mathrm{O}_{4}\right)$ nanoparticles without surfactants at room temperature, Materials Lett. 61 (2007) 4447-4451.

[32] N. Mahmed, O Heczko, O. Söderberg, S.-P. Hannula, Room temperature synthesis of magnetite $\left(\mathrm{Fe}_{3-\delta} \mathrm{O}_{4}\right)$ nanoparticles by a simple reverse co-precipitation method, IOP Conf. Series: Mater. Sci. Eng. 18 (2011) Symposium 2A.

[33] S. Santra, R. Tapec, N. Theodoropoulou, J. Dobson, A. Hebard, W. Tan, Synthesis and Characterization of Silica-Coated Iron Oxide Nanoparticles in Microemulsion: The Effect of Nonionic Surfactants, Langmuir 17 (2001) 2900-2906.

[34] S. Batzri, E. D. Korn, Single bilayer liposomes prepared without sonication, Biochim. Biophys. Acta 298 (1973) 1015-1019. 
[35] J. M. H. Kremer, M. W. J. vd Esker, C. Pathmamanoharan, P. H. Wiersema, Vesicles of variable diameter prepared by a modified injection method, Biochemistry 16 (1977) 39323935 .

[36] Y. Tamba, H. Terashima, M. Yamazaki, A membrane filtering method for the purification of giant unilamellar vesicles, Chem. Phys. Lipids 164 (2011) 351-358.

[37] T. Tanaka, Y. Tamba, S. Md. Masum, Y. Yamashita, M. Yamazaki, $\mathrm{La}^{3+}$ and $\mathrm{Gd}^{3+}$ induce shape change of giant unilamellar vesicles of phosphatidylcholine, Biochim. Biophys. Acta 1564 (2002) 173-182.

[38] B. Valeur, Molecular Fluorescence - Principles and Applications, Wiley-VCH, Weinheim, 2002.

[39] J. N. Demas, G. A. Crosby, Measurement of photoluminescence quantum yields. Review, J. Phys. Chem. 75 (1971) 991-1024.

[40] S. Fery-Forgues, D. Lavabre, Are fluorescence quantum yields so tricky to measure? A demonstration using familiar stationery products, J. Chem. Educ. 76 (1999) 1260-1264.

[41] I. Johnson, M. T. Z. Spence, Molecular Probes Handbook: A Guide to Fluorescent Probes and Labeling Technologies, 11th Ed., Invitrogen, 2011.

[42] A. S. Edelstein, R. C. Cammaratra, Nanomaterials: Synthesis, Properties and Applications, Taylor \& Francis Group, New York, 1996.

[43] M. Tarek, M. Zaki, A. Y. EI-Sayed, Spectrophotometric determination of iron as phenylfluorone complex sensitized with Triton X-100, Fresenius Z. Anal. Chem. 334 (1989) 335-339.

[44] J. Rodriguez-Carvajal, FULLPROF: A Program for Rietveld refinement and pattern matching analysis, Abstracts of the Satellite Meeting on Powder Diffraction of the XV Congress of the IUCr, p. 127, Toulouse, 1990.

[45] W. A. Dollase, Correction of intensities for preferred orientation in powder diffractometry: Application of the March model, J. Appl. Cryst. 19 (1986) 267-272.

[46] L.T. Jule, F. B. Dejene, A. G. Ali, K. T. Roro, A. Hegazy, N. K. Allam, E. El Shenawy, Wide visible emission and narrowing band gap in $\mathrm{Cd}$-doped $\mathrm{ZnO}$ nanopowders synthesized via sol-gel route, J. Alloys Compd. 687 (2016) 920-926.

[47] S. Yildirimcan, K. Ocakoglu, S. Erat, F. M. Emen, S. Repp, E. Erdem, The effect of growing time and $\mathrm{Mn}$ concentration on the defect structure of $\mathrm{ZnO}$ nanocrystals: $\mathrm{X}$-ray diffraction, infrared and EPR spectroscopy, RSC Adv. 6 (2016) 39511-39521.

[48] B. D. Cullity, C. D. Graham, Introduction to Magnetic Materials, John Wiley \& Sons Inc., Hoboken, New Jersey, 2009; ISBN: 9780471477419.

[49] Z. Li-Ying, D. Yong-Hua, Z. Ling, G. Hong-Chen, Magnetic behavior and heating effect of $\mathrm{Fe}_{3} \mathrm{O}_{4}$ ferrofluids composed of monodisperse nanoparticles, Chin. Phys. Lett. 24 (2010) 483-486.

[50] W. Lu, Y. Shen, A. Xie, W. Zhang, Green synthesis and characterization of superparamagnetic $\mathrm{Fe}_{3} \mathrm{O}_{4}$ nanoparticles, J. Magn. Magn. Mater. 322 (2010) 1828-1833.

[51] C. Hui, C. Shen, T. Yang, L. Bao, J. Tian, H. Ding, C. Li, H.-J. Gao, Large-scale $\mathrm{Fe}_{3} \mathrm{O}_{4}$ nanoparticles soluble in water synthesized by a facile method, J. Phys. Chem. C 112 (2008) 11336-11339. 
[52] S. Zhao, S. Asuha, One-pot synthesis of magnetite nanopowder and their magnetic properties, Powder Technol. 197 (2010) 295-297.

[53] W. Pei, H. Kumada, T. Natusme, H. Saito, S. Ishio, Study on magnetite nanoparticles synthesized by chemical method, J. Magn. Magn. Mater. 310 (2007) 2375-2377.

[54] K. Parekh, R. V. Upadhyay, V. K. Aswal, Monodispersed superparamagnetic $\mathrm{Fe}_{3} \mathrm{O}_{4}$ nanoparticles: synthesis and characterization, J. Nanosci. Nanotechnol., 9 (2009) 21042110.

[55] D.-L. Zhao, X.-W. Zeng, Q.-S. Xia, J.-T. Tang, Preparation and coercivity and saturation magnetization dependence of inductive heating property of $\mathrm{Fe}_{3} \mathrm{O}_{4}$ nanoparticles in an alternating current magnetic field for localized hyperthermia, J. Alloys Compd. 469 (2009) 215-218.

[56] L. Blaney, Magnetite $\left(\mathrm{Fe}_{3} \mathrm{O}_{4}\right)$ : Properties, Synthesis, and Applications, Lehigh Review, 15 (2007) article 5.

[57] J. Pearce, A. Giustini, R. Stigliano, P. J. Hoopes, Magnetic Heating of Nanoparticles: The Importance of Particle Clustering to Achieve Therapeutic Temperatures, J. Nanotechnol. Eng. Med. 4 (2013) article 011005.

[58] R. Sabaté, R. Barnadas-Rodríguez, J. Callejas-Fernández, R. Hidalgo-Alvarez, J. Estelrich, Preparation and characterization of extruded magnetoliposomes, Int. J. Pharm. 347 (2008) 156-162.

[59] R. V. Ferreira, T. M. M. Martins, A. M. Goes, J. D. Fabris, L. C. D. Cavalcante, L. E. F. Outon, R. Z. Domingues, Thermosensitive gemcitabine-magnetoliposomes for combined hyperthermia and chemotherapy, Nanotechnology 27 (2016) 085105.

[60] A. R. O. Rodrigues, I. T. Gomes, B. G. Almeida, J. P. Araújo, E. M. S. Castanheira, P. J. G. Coutinho, Magnetoliposomes based on nickel/silica core/shell nanoparticles: synthesis and characterization, Mat. Chem. Phys. 148 (2014) 978-987.

[61] D. K. Struck, D. Hoekstra, R. E. Pagano, Use of resonance energy transfer to monitor membrane fusion, Biochemistry 20 (1981) 4093-4099.

[62] P. Greenspan, S.D. Fowler, Spectrofluorometric studies of the lipid probe, nile red, J. Lipid Res. 26 (1985) 781-789.

[63] I. and G. Krishnamoorthy, Probing the link between proton transport and water content in lipid membranes, J. Phys. Chem. B 105 (2001) 1484-1488.

[64] P. J. G. Coutinho, E. M. S. Castanheira, M. C. Rei, M. E. C. D. Real Oliveira, Nile red and DCM fluorescence anisotropy studies in $\mathrm{C}_{12} \mathrm{E}_{7} / \mathrm{DPPC}$ mixed systems, J. Phys. Chem. B 106 (2002) 12841-12486.

[65] G. Hungerford, E. M. S. Castanheira, M. E. C. D. Real Oliveira, M. G. Miguel, H. D. Burrows, Monitoring ternary systems of $\mathrm{C}_{12} \mathrm{E}_{5} /$ water/tetradecane via the fluorescence of solvatochromic probes, J. Phys. Chem. B 106 (2002) 4061-4069.

[66] B. R. Lentz, Membrane "fluidity" as detected by diphenylhexatriene probes, Chem. Phys. Lipids 50 (1989) 171-190.

[67] H. Curtis, N. Barnes, Biology, $5^{\text {th }}$ Edition, Worth Publishers, New York, 1989.

[68] M. De Cuyper, M. Joniau, Magnetoliposomes. Formation and structural characterization. Eur. Biophys. J. 15 (1988) 311-319. 
[69] D. R. Lide, Ed., CRC Handbook of Chemistry and Physics, 89th Ed., CRC Press/Taylor and Francis, Boca Raton, FL, USA, 2009.

[70] J. N. Israelachvili, S. Marcelja, R. G. Horn, Physical principles of membrane organization, Q. Rev. Biophys. 13 (1980) 121-200.

[71] D. B. Kell, C. M. Harris, On the dielectrically observable consequences of the diffusional motions of lipids and proteins in membranes. 1. Theory and overview, Eur. Biophys. J. 12 (1985) 181-197.

[72] R. Veldhuizen, K. Nag, S. Orgeig, F. Possmayer, The role of lipids in pulmonary surfactant, Biochim. Biophys. Acta 1408 (1998) 90-108.

[73] D. Papahadjopoulos, N. Miller, Phospholipid model membranes. I. Structural characteristics of hydrated liquid crystals, Biochim. Biophys. Acta 135 (1967) 624-638.

[74] L. Tilley, K. R. Thulborn, W. H. Sawyer, An assessment of the fluidity gradient of the lipid bilayer as determined by a set of $n$-(9-anthroyloxy)fatty acids $(n=2,6,9,12,16), \mathrm{J}$. Biol. Chem. 254 (1979) 2592-2594.

[75] M. A. Bahri, B. J. Heyne, P. Hans, A. E. Seret, A. A. Mouithys-Mickalad, M. D. Hoebeke, Quantification of lipid bilayer effective microviscosity and fluidity effect induced by propofol, Biophys. Chem. 114 (2005) 53-61. 